\title{
Stability Analysis of an SIRI Epidemic Model with Distributed Latent Period
}

\author{
Amine Bernoussi ${ }^{1 *}$, Abdelilah Kaddar $^{2}$ and Said Asserda ${ }^{1}$ \\ ${ }^{1}$ Université Ibn Tofail, Faculté des Sciences, Département de Mathématiques, Kénitra, Morocco \\ ${ }^{2}$ Université Mohammed V de Rabat, Faculté des Sciences Juridiques, Economiques et Sociales - Salé, Morocco \\ Email: amine.bernoussi@yahoo.fr
}

\begin{abstract}
In this paper, we investigate the global asymptotic stability of the possible equilibria for an SIRI epidemic model with a distributed latent period and a general incidence function. By using the method of Lyapunov functions and the LaSalle invariance principle, we show that the global asymptotic stability is completely determined by the basic reproduction number $R_{0}$. The originality of this work is to have a basic reproduction number $R_{0}$ which depends on the distribution of the latent period. Finally, we use a nonstandard discretization method of Mickens to illustrate numerically our theoretical results.
\end{abstract}

Keywords: Epidemic model, distributed time delay, Lyapunov function, global stability

\section{Introduction}

The epidemic models with time delay have long been an important subject for the mathematical researchers (see $[1,2,3,4,5,6]$ for a discrete delay and $[7,8]$ for a distributed delay), because many diseases, such as Influenza, Ébola and Tuberculosis, have a latent period, during which the individual is said to be exposed but not infectious. Therefore, it is important to ask the following question: how is it possible to model the latent period in order to improve the control of the spread of diseases?

In most statistical researches, motivated by this question, the latent period distribution may take different forms:

- The first one is a gamma distribution. For example, the latent period distribution of the avian influenza was modeled by a gamma distribution in agreement with the experiments [9].

- The second one is a log-normally distribution. For example, the latent period distribution of ebola was estimated to be log-normally distributed [10].

In this paper, we propose the following SIRI epidemic model with a distributed time delay and a relapse phenomenon, i.e. the return of signs and symptoms of a disease after a remission $[11,12,13,14,6]$ :

$$
\left\{\begin{array}{c}
\frac{\mathrm{d} S}{\mathrm{~d} t}=A-\mu S-f(S, I) \\
\frac{\mathrm{d} I}{\mathrm{~d} t}=\int_{0}^{h} p(\tau) e^{-\mu \tau} f\left(S_{\tau}, I_{\tau}\right) \mathrm{d} \tau-(\mu+\gamma+\alpha) I+\delta R \\
\frac{\mathrm{d} R}{\mathrm{~d} t}=\gamma I-(\mu+\delta) R .
\end{array}\right.
$$

The initial condition for the above system is

$$
S(\theta)=\varphi_{1}(\theta), \quad I(\theta)=\varphi_{2}(\theta), \quad R(\theta)=\varphi_{3}(\theta), \quad \theta \in[-h, 0]
$$

with $\varphi=\left(\varphi_{1}, \varphi_{2}, \varphi_{3}\right) \in C^{+} \times C^{+} \times C^{+}$, such that $\varphi_{i}(\theta) \geq 0(-\tau \leq \theta \leq 0, i=1,2,3)$.

Here $C$ denotes the Banach space $C([-h, 0], \mathbb{R})$ of continuous functions mapping the interval $[-h, 0]$ into $\mathbb{R}$, equipped with the supremum norm. The non-negative cone of $C$ is defined as $C^{+}=C\left([-h, 0], \mathbb{R}^{+}\right)$, where $\mathbb{R}^{+}=\{x \in \mathbb{R} \mid x \geq 0\}$. 
Here $\psi_{\tau}=\psi(t-\tau)$ for any given function $\psi, A=\mu N$, where $N$ is the total number of population, $S$ is the number of susceptible individuals, $I$ is the number of infectious individuals, $\mu$ denotes birth and death rates, $\alpha$ denotes death rate due to the disease, $f(S, I)$ is the incidence function, i.e. the number of susceptible individuals infected through their contacts with the infectious individuals, $\gamma$ is the recovery rate of the infectious individuals, $\delta$ represents the rate that recovered individuals relapse and regain the infectious class, $p(\tau)$ is the latent period distribution, which denotes the fraction of individuals who become infective $\tau$ units of time after infection and $h$ is a maximum time taken by infected individual to become able to transmit the infection (maximum latent period).

In model (1) the incidence function $f(S, I)$ is a locally Lipschitz continuous function on $\mathbb{R}^{+} \times \mathbb{R}^{+}$ satisfaying $f(0, I)=f(S, 0)=0$ for $S \geq 0, I \geq 0$ and the followings hold:

$\left(H_{1}\right) f$ is a strictly monotone increasing function of $S \geq 0$, for any fixed $I>0$, and $f$ is a strictly monotone increasing function of $I \geq 0$, for any fixed $S \geq 0$;

$\left(H_{2}\right) \phi(S, I)=\frac{f(S, I)}{I}$ is a bounded and monotone decreasing function of $I>0$, for any fixed $S \geq 0$, and $K(S)=\lim _{I \rightarrow 0^{+}} \phi(S, I)$ is a continuous and monotone increasing function on $S \geq 0$.

This incidence function includes the following forms: The first one is the saturated incidence $\frac{\beta S I}{d+S+I}$ [15], where $\beta$ and $d$ are the positive constants. The second one is the bilinear incidence $\beta S I[16,17,18,19,20]$. The third one is the saturated incidence $\frac{\beta S I}{1+\alpha_{1} S+\alpha_{2} I}[15,21,22,23,17,24,25,26]$, where $\alpha_{1}$ and $\alpha_{2}$ are the positive constants. The effect of the saturation factor (refer to $\alpha_{1}$ and $\alpha_{2}$ ) stems from epidemic control and the protection measures. The fourth one is the standard incidence $\frac{\beta S I}{N}[27,28]$.

Historically, Tudor [13] developed and analyzed qualitatively one of the first SIRI epidemic model for the spread of a herpes-type infection in either human or animal populations. This model consists of a system of non-linear ordinary differential equations with a bilinear incidence function and a constant total population. Based on the Tudor model, Moreira and Wang [29] extended a Tudor-model to include non-linear incidence functions and they derived sufficient conditions for the global asymptotic stability of the disease-free and endemic equilibria (see also [30,31,32,33,34] for particular incidence functions).

In [35,36], Van den Driessche et al. proposed an integro-differential equation to model a general relapse phenomenon in infectious diseases (see also [34,37]). The resulting model, in particular case, is a differential equation with discrete time delay, a constant population and a particular incidence functions (standard and bilinear). The basic reproduction number for this model is identified and some global results are obtained and the disease is shown to be uniformly persistent with the infective population size either approaching or oscillating about the endemic level. The effect of the discrete time delay in the epidemic models with a relapse rate has been taken into account in some recent studies ( see, for example $[6,38,39])$.

As observed in empirical data [9,10], the researchers modeled the latent period by introducing a distributed time delay in epidemic models and they demonstrated that distributed delay has the same effect as the discrete time delay (the basic reproduction number is independent on the distribution of the latent period). This implies that the distribution of latent period has no effect on the spread of infectious diseases $[40,41,42]$.

In this paper we extend the global stability results presented in $[6,38]$ to an SIRI epidemic model with a distributed time delay and a general incidence function. It is established that the basic reproduction number $R_{0}$ depends on the distribution of the latent period, the incidence function and the relapse rate and it is shown that the global stability can be attained under suitable sufficient conditions on the incidence function and the threshold parameter $R_{0}$. The originality of this work is to introduce both a distributed time delay, a general incidence function and a relapse rate in the SIR epidemic model (see model (1)).

The rest of the paper is organized as follows. In Section 2, we establish the global stability of diseasefree and endemic equilibria. Section 3 gives a numerical analysis and concluding remarks concerning a comparison of a distributed SIRI model with a discrete SIRI model, and finally, in the section 4 , we include our conclusions and our future researches. 


\section{Global Stability Analysis of Delayed SIRI Model}

System (1) always has a disease-free equilibrium $P_{0}=\left(\frac{A}{\mu}, 0,0\right)$. On the other hand, under the hypotheses $\left(H_{1}\right)$ and $\left(H_{2}\right)$, if

$$
R_{0}:=\frac{K\left(\frac{A}{\mu}\right) \kappa(h)}{\eta}>1,
$$

then system (1) also admits a unique endemic equilibrium $P^{*}=\left(S^{*}, I^{*}, R^{*}\right)$, where $S^{*}, I^{*}$ and $R^{*}$ satisfy the following system:

$$
\left\{\begin{array}{c}
A-\mu S-f(S, I)=0 \\
k(h) f(S, I)-(\mu+\gamma+\alpha) I+\delta R=0, \\
\gamma I-(\mu+\delta) R=0
\end{array}\right.
$$

with $\kappa(h)=\int_{0}^{h} p(\tau) e^{-\mu \tau} \mathrm{d} \tau$ and $\eta=\mu+\gamma+\alpha-\frac{\gamma \delta}{\mu+\delta}$.

Remark 1. The obtained basic reproduction number (3) depends on the distribution of the latent period $\left(\kappa(h)=\int_{0}^{h} p(\tau) e^{-\mu \tau} \mathrm{d} \tau\right)$. In the literature, there is no formula similar to (3).

In this section, we will discuss the global stability of the disease-free equilibrium $P_{0}$ and the endemic equilibrium $P^{*}$ of system (1). Since $\frac{d}{\mathrm{~d} t}(S+I+R) \leq A-\mu(S+I+R)$, we have $\lim \sup (S+I+R) \leq \frac{A}{\mu}$. Hence we discuss system (1) in the closed set

$$
\Omega=:\left\{\left(\varphi_{1}, \varphi_{2}, \varphi_{3}\right) \in C^{+} \times C^{+} \times C^{+}:\left\|\varphi_{1}+\varphi_{2}+\varphi_{3}\right\| \leq A / \mu\right\} .
$$

It is easy to show that $\Omega$ is positively invariant with respect to system (1). Next we consider the global asymptotic stability of the disease-free equilibrium $P_{0}$ and the endemic equilibrium $P^{*}$ of $(1)$ by Lyapunov's functions, respectively.

Proposition 2. If $R_{0} \leq 1$, then the disease-free equilibrium $P_{0}$ is globally asymptotically stable.

Proof. Consider the following Lyapunov functional

$$
\begin{aligned}
V_{0}(t)= & \int_{0}^{h} p(\tau) e^{-\mu \tau} \int_{\frac{A}{\mu}}^{S_{\tau}}\left(1-\frac{K\left(\frac{A}{\mu}\right)}{K(u)}\right) \mathrm{d} u \mathrm{~d} \tau+I+\frac{\delta}{\mu+\delta} R \\
& +\int_{0}^{h} p(\tau) e^{-\mu \tau} \int_{t-\tau}^{t} \frac{K\left(\frac{A}{\mu}\right)}{K(S(u))} f(S(u), I(u)) \mathrm{d} u \mathrm{~d} \tau .
\end{aligned}
$$

We will show that $\frac{\mathrm{d} V_{0}(t)}{\mathrm{d} t} \leq 0$ for all $t \geq 0$. We have

$$
\begin{aligned}
\frac{\mathrm{d} V_{0}(t)}{\mathrm{d} t}= & \mu \int_{0}^{h} p(\tau) e^{-\mu \tau}\left(1-\frac{K\left(\frac{A}{\mu}\right)}{K\left(S_{\tau}\right)}\right)\left(\frac{A}{\mu}-S_{\tau}\right) \mathrm{d} \tau \\
& +\int_{0}^{h} p(\tau) e^{-\mu \tau} \frac{K\left(\frac{A}{\mu}\right)}{K(S)} f(S, I) \mathrm{d} \tau-\eta I .
\end{aligned}
$$

Furthermore, it follows from the hypothesis $\left(\mathrm{H}_{2}\right)$ that

$$
\begin{aligned}
\int_{0}^{h} p(\tau) e^{-\mu \tau} \frac{K\left(\frac{A}{\mu}\right)}{K(S)} f(S, I) \mathrm{d} \tau & \leq \int_{0}^{h} p(\tau) e^{-\mu \tau} \frac{K\left(\frac{A}{\mu}\right)}{K(S)} \phi(S, I) I \mathrm{~d} \tau \\
& \leq \eta I R_{0} .
\end{aligned}
$$

By the hypothesis $\left(H_{2}\right)$, we obtain that

$$
\left(1-\frac{K\left(\frac{A}{\mu}\right)}{K\left(S_{\tau}\right)}\right)\left(\frac{A}{\mu}-S_{\tau}\right) \leq 0,
$$


where equality holds if and only if $S=\frac{A}{\mu}$.

Therefore, $R_{0} \leq 1$ ensures that $\frac{\mathrm{d} V_{0}(t)}{\mathrm{d} t} \leq 0$ for all $t \geq 0$, where $\frac{\mathrm{d} V_{0}(t)}{\mathrm{d} t}=0$ holds if $(S, I, R)=\left(\frac{A}{\mu}, 0,0\right)$. Hence, it follows from system (1) that $\left\{P_{0}\right\}$ is the largest invariant set in $\left\{(S, I, R) \mid \frac{\mathrm{d} V_{0}(t)}{\mathrm{d} t}=0\right\}$. From the Lyapunov-LaSalle asymptotic stability, we obtain that $P_{0}$ is globally asymptotically stable. This completes the proof.

Proposition 3. If $R_{0}>1$, then the endemic equilibrium $P^{*}$ is globally asymptotically stable.

Proof. Firstly, we prove the existence and the uniqueness of the endemic equilibrium $P^{*}$. At a fixed point $(S, I, R)$ of system (1), the following equations hold.

$$
\left\{\begin{array}{c}
A-\mu S-f(S, I)=0, \\
\kappa(h) f(S, I)-(\mu+\gamma+\alpha) I+\delta R=0, \\
\gamma I-(\mu+\delta) R=0 .
\end{array}\right.
$$

Substituting the third equation into the second equation of (5), we consider the following system:

$$
\left\{\begin{array}{c}
A-\mu S-f(S, I)=0, \\
\kappa(h) f(S, I)-\left(\mu+\gamma+\alpha-\frac{\gamma \delta}{\mu+\delta}\right) I=0, \\
R=\frac{\gamma I}{\mu+\delta} .
\end{array}\right\}
$$

Using the first and the second equations in (6), we conclude that

$$
S=\frac{A}{\mu}-\left(\mu+\gamma+\alpha-\frac{\delta \gamma}{\mu+\delta}\right) \frac{I}{\mu \kappa(h)} .
$$

Substituting the equation (7) into the second equation of (5), we have

$$
g(I):=\frac{f\left(\frac{A}{\mu}-\left(\mu+\gamma+\alpha-\frac{\delta \gamma}{\mu+\delta}\right) \frac{I}{\mu \kappa(h)}, I\right)}{I}-\frac{\mu+\gamma+\alpha-\frac{\gamma \delta}{\mu+\delta}}{\kappa(h)}=0 .
$$

By the hypothesis $\left(H_{2}\right), g$ is strictly monotone decreasing on $\left.] 0, \frac{A \kappa(h)}{\left(\mu+\gamma+\alpha-\frac{\gamma \delta}{\mu+\delta}\right)}\right]$ satisfying:

$$
\lim _{I \rightarrow 0^{+}} g(I)=\frac{\left(\mu+\gamma+\alpha-\frac{\gamma \delta}{\mu+\delta}\right)}{\kappa(h)}\left(R_{0}-1\right)>0,
$$

and

$$
g\left(\frac{A \kappa(h)}{\left(\mu+\gamma+\alpha-\frac{\gamma \delta}{\mu+\delta}\right)}\right)=-\frac{\left(\mu+\gamma+\alpha-\frac{\gamma \delta}{\mu+\delta}\right)}{\kappa(h)}<0 .
$$

Thus, there exists a unique $I^{*}$ such that $g\left(I^{*}\right)=0$. Hence, we conclude the existence and uniqueness of the endemic equilibrium $P^{*}$.

Finally, To prove global stability of the endemic equilibrium, we define a Lyapunov functional

$$
V(t)=V_{1}(t)+V_{2}(t)+V_{3}(t)+V_{+},
$$

with

$$
V_{1}(t)=\int_{0}^{h} p(\tau) e^{-\mu \tau} \mathrm{d} \tau\left(S-S^{*}-\int_{S^{*}}^{S} \frac{f\left(S^{*}, I^{*}\right)}{f\left(u, I^{*}\right)} \mathrm{d} u\right),
$$

and

$$
V_{2}(t)=\left(I-I^{*}-I^{*} \ln \frac{I}{I^{*}}\right),
$$




$$
V_{3}(t)=\frac{\delta}{\mu+\delta}\left(R-R^{*}-R^{*} \ln \frac{R}{R^{*}}\right)
$$

and

$$
V_{+}=\int_{0}^{h} \int_{t-\tau}^{t} p(\tau) e^{-\mu \tau}\left[\left(f(S, I)-f\left(S^{*}, I^{*}\right)-f\left(S^{*}, I^{*}\right) \ln \frac{f(S(u), I(u))}{f\left(S^{*}, I^{*}\right)}\right)\right] \mathrm{d} u \mathrm{~d} \tau
$$

The time derivative of the function $V(t)$ along the positive solution of system (1) is

$$
\begin{aligned}
\frac{\mathrm{d} V(t)}{\mathrm{d} t}= & \int_{0}^{h}\left(p(\tau) e^{-\mu \tau}\right) \mathrm{d} \tau\left(1-\frac{f\left(S^{*}, I^{*}\right)}{f\left(S, I^{*}\right)}\right)(A-\mu S-f(S, I)) \\
& +\left(1-\frac{I^{*}}{I}\right)\left(\int_{0}^{h} p(\tau) e^{-\mu \tau} f\left(S_{\tau}, I_{\tau}\right) \mathrm{d} \tau+\delta R-(\mu+\alpha+\gamma) I\right) \\
& +\frac{\delta}{\mu+\delta}\left(1-\frac{R^{*}}{R}\right)(\gamma I-(\mu+\delta) R) \\
& +\int_{0}^{h}\left(p(\tau) e^{-\mu \tau}\right)\left[f(S, I)-f\left(S_{\tau}, I_{\tau}\right)+f\left(S^{*}, I^{*}\right) \ln \frac{f\left(S_{\tau}, I_{\tau}\right)}{f(S, I)}\right] \mathrm{d} \tau
\end{aligned}
$$

Using the relation $A=\mu S^{*}+f\left(S^{*}, I^{*}\right)$, simple calculations give that

$$
\begin{aligned}
\frac{\mathrm{d} V(t)}{\mathrm{d} t}= & \int_{0}^{h}\left(p(\tau) e^{-\mu \tau}\right) \mathrm{d} \tau\left[\left(1-\frac{f\left(S^{*}, I^{*}\right)}{f\left(S, I^{*}\right)}\right)\left(-\mu\left(S-S^{*}\right)+f\left(S^{*}, I^{*}\right)\right)\right] \\
& +\int_{0}^{h}\left(p(\tau) e^{-\mu \tau}\right) \mathrm{d} \tau \frac{f\left(S^{*}, I^{*}\right)}{f\left(S, I^{*}\right)} f(S, I) \\
& -(\mu+\alpha+\gamma) I-e^{\mu \tau} \frac{I^{*}}{I} \int_{0}^{h}\left(p(\tau) e^{-\mu \tau}\right) f\left(S_{\tau}, I_{\tau}\right) \mathrm{d} \tau-\delta \frac{I^{*}}{I} R+(\mu+\alpha+\gamma) I^{*} \\
& +\frac{\delta}{\mu+\delta}\left(\gamma I-\gamma I \frac{R^{*}}{R}+(\mu+\delta) R^{*}\right) \\
& +\int_{0}^{h}\left(p(\tau) e^{-\mu \tau}\right) f\left(S^{*}, I^{*}\right) \ln \frac{f\left(S_{\tau}, I_{\tau}\right)}{f(S, I)} \mathrm{d} \tau
\end{aligned}
$$

Here by using

$$
\begin{gathered}
(\mu+\alpha+\gamma) I^{*}-\frac{\delta}{\mu+\delta} \gamma I^{*}=f\left(S^{*}, I^{*}\right) \int_{0}^{h} p(\tau) e^{-\mu \tau} \mathrm{d} \tau, \\
(\mu+\delta) R^{*}=\gamma I^{*},
\end{gathered}
$$

and

$$
\ln \frac{f\left(S_{\tau}, I_{\tau}\right)}{f(S, I)}=\ln \frac{f\left(S^{*}, I^{*}\right)}{f\left(S, I^{*}\right)}+\ln \frac{I^{*} f\left(S_{\tau}, I_{\tau}\right)}{I f\left(S^{*}, I^{*}\right)}+\ln \frac{I f\left(S, I^{*}\right)}{I^{*} f(S, I)}
$$


straightforward calculations give

$$
\begin{aligned}
& \frac{\mathrm{d} V(t)}{\mathrm{d} t}=-\mu \int_{0}^{h}\left(p(\tau) e^{-\mu \tau}\right) \mathrm{d} \tau\left(1-\frac{f\left(S^{*}, I^{*}\right)}{f\left(S, I^{*}\right)}\right)\left(S-S^{*}\right) \\
& -\int_{0}^{h}\left(p(\tau) e^{-\mu \tau}\right) \mathrm{d} \tau f\left(S^{*}, I^{*}\right)\left(\frac{f\left(S^{*}, I^{*}\right)}{f\left(S, I^{*}\right)}-1-\ln \frac{f\left(S^{*}, I^{*}\right)}{f\left(S, I^{*}\right)}\right) \\
& -f\left(S^{*}, I^{*}\right) \int_{0}^{h}\left(p(\tau) e^{-\mu \tau}\right)\left(\frac{I^{*} f\left(S_{\tau}, I_{\tau}\right)}{I f\left(S^{*}, I^{*}\right)}-1-\ln \frac{I^{*} f\left(S_{\tau}, I_{\tau}\right)}{I f\left(S^{*}, I^{*}\right)}\right) \mathrm{d} \tau \\
& +f\left(S^{*}, I^{*}\right) \int_{0}^{h}\left(p(\tau) e^{-\mu \tau}\right) \mathrm{d} \tau\left(\frac{f(S, I)}{f\left(S, I^{*}\right)}-\frac{I}{I^{*}}+\frac{I f\left(S, I^{*}\right)}{I^{*} f(S, I)}-1\right) \\
& -f\left(S^{*}, I^{*}\right) \int_{0}^{h}\left(p(\tau) e^{-\mu \tau}\right) \mathrm{d} \tau\left(\frac{I f\left(S, I^{*}\right)}{I^{*} f(S, I)}-1-\ln \frac{I f\left(S, I^{*}\right)}{I^{*} f(S, I)}\right) \\
& +\frac{\delta \gamma I^{*}}{\mu+\delta}\left(2-\frac{I^{*} R}{I R^{*}}-\frac{I R^{*}}{I^{*} R}\right) \\
& =-\mu \int_{0}^{h}\left(p(\tau) e^{-\mu \tau}\right) \mathrm{d} \tau\left(1-\frac{f\left(S^{*}, I^{*}\right)}{f\left(S, I^{*}\right)}\right)\left(S-S^{*}\right) \\
& -\int_{0}^{h}\left(p(\tau) e^{-\mu \tau}\right) \mathrm{d} \tau f\left(S^{*}, I^{*}\right)\left(\frac{f\left(S^{*}, I^{*}\right)}{f\left(S, I^{*}\right)}-1-\ln \frac{f\left(S^{*}, I^{*}\right)}{f\left(S, I^{*}\right)}\right) \\
& -f\left(S^{*}, I^{*}\right) \int_{0}^{h}\left(p(\tau) e^{-\mu \tau}\right)\left(\frac{I^{*} f\left(S_{\tau}, I_{\tau}\right)}{I f\left(S^{*}, I^{*}\right)}-1-\ln \frac{I^{*} f\left(S_{\tau}, I_{\tau}\right)}{I f\left(S^{*}, I^{*}\right)}\right) \mathrm{d} \tau \\
& -f\left(S^{*}, I^{*}\right) \int_{0}^{h}\left(p(\tau) e^{-\mu \tau}\right) \mathrm{d} \tau\left(1-\frac{f(S, I)}{f\left(S, I^{*}\right)}\right)\left(1-\frac{\phi\left(S, I^{*}\right)}{\phi(S, I)}\right) \\
& -f\left(S^{*}, I^{*}\right) \int_{0}^{h}\left(p(\tau) e^{-\mu \tau}\right) \mathrm{d} \tau\left(\frac{I f\left(S, I^{*}\right)}{I^{*} f(S, I)}-1-\ln \frac{I f\left(S, I^{*}\right)}{I^{*} f(S, I)}\right) \\
& -\frac{\delta \gamma}{\mu+\delta} \frac{I R^{*}}{R}\left(\frac{I^{*} R}{I R^{*}}-1\right)^{2}
\end{aligned}
$$

It follows from $\left(H_{1}\right)$ and $\left(H_{2}\right)$ that

$$
-\mu\left(1-\frac{f\left(S^{*}, I^{*}\right)}{f\left(S, I^{*}\right)}\right)\left(S-S^{*}\right) \leq 0,
$$

and

$$
-f\left(S^{*}, I^{*}\right)\left(1-\frac{f(S, I)}{f\left(S, I^{*}\right)}\right)\left(1-\frac{\phi\left(S, I^{*}\right)}{\phi(S, I)}\right) \leq 0 .
$$

Furthermore, since the function $g(x)=1-x+\ln (x)$ is always non-positive for any $x>0$, and $g(x)=0$ if and only if $x=1$, then $\frac{\mathrm{d} V(t)}{\mathrm{d} t} \leq 0$, for all $t \geq 0$, where the equality holds only at the equilibrium point $\left(S^{*}, I^{*}, R^{*}\right)$. Hence, $P^{*}$ is globally asymptotically stable.

Remark 4. In Proposition 3, by modifying Lyapunov function proposed in [6], we proved that the endemic equilibrium is globally asymptotically stable. The obtained result extended the Theorem (3.1) in [38]. But the Lyapunov function proposed in [38] is not valid for our model (1).

\section{$3 \quad$ Numerical Simulations}

In the following subsections, we will numerically indicate the risk of using the discrete or distributed time delay to describe the latent period. Also, we propose to illustrate graphically the effect of the relapse phenomenon on the basic reproduction number $R_{0}$.

First, in some cases the SIRI epidemic model with discrete delay and its corresponding version with distributed delay generate the same asymptotic behavior. However, we note significant differences in 
the speed of convergence and the evolution of the infected individuals (see subsection 3.1). Second, we compared the basic reproduction number $R_{0}$ of both models to bring out another major difference: the two models do not have the same equilibrium (see subsection 3.2). Finally, we illustrate the effect of the rate of relapse on the reproduction number $R_{0}$ and we observe that the relapse phenomenon increases the risk of installation of the disease in the considered population (see subsection 3.3).

\subsection{Effect of the Latent Period on the Asymptotic Behavior}

In this subsection, we give a numerical simulation to illustrate the theoretical analysis given in section 2 and to compare a distributed SIRI model (1) with the following discrete SIRI model (SIRI model with constant time delay) [6]:

$$
\left\{\begin{array}{c}
\frac{\mathrm{d} S}{\mathrm{~d} t}=A-\mu S-f(S, I) \\
\frac{\mathrm{d} I}{\mathrm{~d} t}=e^{-\mu h} f\left(S_{h}, I_{h}\right)-(\mu+\gamma+\alpha) I+\delta R \\
\frac{\mathrm{d} R}{\mathrm{~d} t}=\gamma I-(\mu+\delta) R
\end{array}\right\}
$$

Let

$$
f(S, I)=\beta S I \quad \text { and } \quad p(\tau)=\frac{1}{h}
$$

Applying Mickens nonstandard discretization (see, $[43,44,45])$ to the continuous model (1), we first derive the following discretized SIRI epidemic model with a distributed time delay:

$$
\left\{\begin{array}{c}
S_{n+1}-S_{n}=A-\mu S_{n+1}-\beta S_{n+1} I_{n+1}, \\
I_{n+1}-I_{n}=\frac{\beta}{h} \sum_{k=0}^{h} e^{-\mu k} S_{n-k} I_{n-k}-(\mu+\gamma+\alpha) I_{n+1}+\delta R_{n+1}, \\
R_{n+1}-R_{n}=\gamma I_{n+1}-(\mu+\delta) R_{n+1}, n=0,1,2, \ldots
\end{array}\right\}
$$

The initial conditions of the system (12) are given by

$$
S_{n}=\psi_{n}^{(1)}, I_{n}=\psi_{n}^{(2)}, R_{n}=\psi_{n}^{(3)}, \text { for }, n=-h,-h+1, \ldots, 0,
$$

where $\psi_{n}^{(i)} \geq 0(n=-h,-h+1, \ldots, 0, i=1,2,3)$. We further assume that $\psi_{0}^{(i)}>0$ for $i=1,2,3$.

Substituting the third equation into the second equation of (12), we consider the following system:

$$
\left\{\begin{array}{c}
S_{n+1}=\frac{A+S_{n}}{1+\mu+\beta I_{n+1}}, \\
I_{n+1}=\frac{\beta}{(1+c) h} \sum_{k=0}^{h} e^{-\mu k} S_{n-k} I_{n-k}+\frac{I_{n}}{1+c}+c_{1} R_{n}, \\
R_{n+1}=\frac{\gamma}{1+(\mu+\delta)} I_{n+1}+\frac{R_{n}}{1+(\mu+\delta)},
\end{array}\right\}
$$

with

$$
c=\frac{(\mu+\gamma+\alpha)+(\mu+\delta)(\mu+\alpha)+\mu \gamma}{1+(\mu+\delta)} \text { and } c_{1}=\frac{\delta}{1+(\mu+\delta)} \frac{1}{(1+c)}
$$

Therefore we have 


$$
\left\{\begin{array}{c}
S_{n+1}=\frac{A+S_{n}}{1+\mu+\beta\left[\frac{\beta}{(1+c) h} \sum_{k=0}^{h} e^{-\mu k} S_{n-k} I_{n-k}+\frac{I_{n}}{1+c}+c_{1} R_{n}\right]}, \\
I_{n+1}=\frac{\beta}{(1+c) h} \sum_{k=0}^{h} e^{-\mu k} S_{n-k} I_{n-k}+\frac{I_{n}}{1+c}+c_{1} R_{n}, \\
R_{n+1}=\frac{\gamma}{1+(\mu+\delta)}\left[\frac{\beta}{(1+c) h} \sum_{k=0}^{h} e^{-\mu k} S_{n-k} I_{n-k}+\frac{I_{n}}{1+c}+c_{1} R_{n}\right]+\frac{R_{n}}{1+(\mu+\delta)} .
\end{array}\right\}
$$

We take the parameters of the system (1) as follows:

$$
\begin{gathered}
A=5, \quad \mu=0.005, \quad \gamma=0.02, \quad \alpha=0.005, \\
\beta=0.1, \quad \delta=0.001, \quad h=10, \quad S(0)=999, \quad I(0)=1, \quad R(0)=0 .
\end{gathered}
$$

By Proposition 3, the endemic equilibrium $P^{*}$ of the distributed SIRI model (1) is globally asymptotically stable (see Fig. 1). In addition, the models (1) and (11) generate identical, global asymptotic behavior with different rates of convergence: For example, the maximum of infectious individuals is achieved, for the model (1), at time $t=500$ days ( 1 years and 4 months), but this maximum is reached, for the model (11), before 200 days (see Figure 1 and Figure 2).
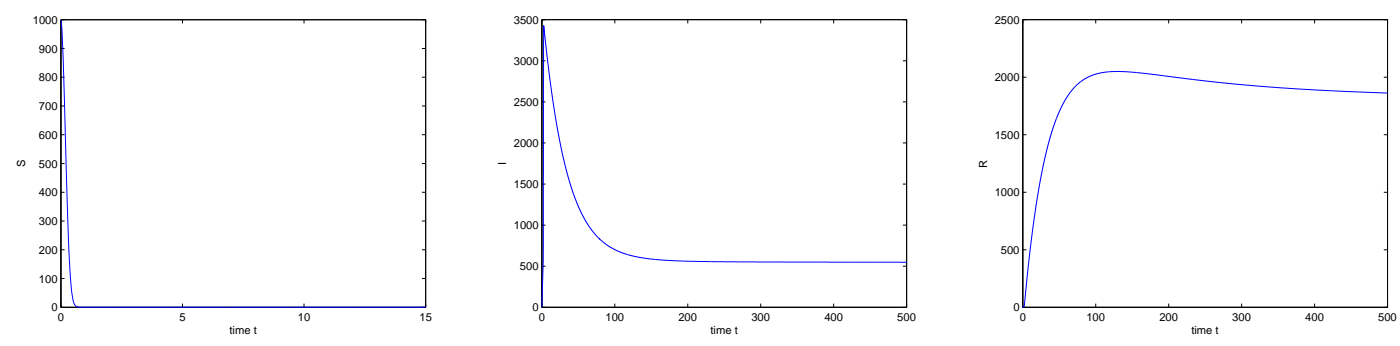

Figure 1. Solutions $(S, I, R)$ of the distributed SIRI epidemic model (1) are globally asymptotically stable and converge to the endemic equilibrium $P^{*}$.
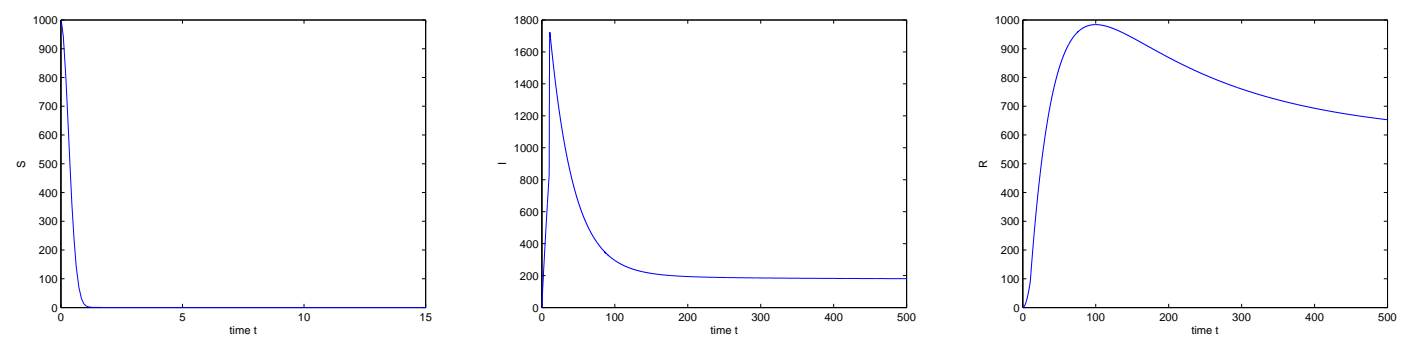

Figure 2. Solutions $(S, I, R)$ of a discrete SIRI epidemic model (11) are globally asymptotically stable and converge to the endemic equilibrium $P^{*}$ 


\subsection{Effect of the Latent Period on the Basic Reproduction Number}

Now, we will show how the basic reproduction number $R_{0}$ changes depending on the latent period. In table 1 , we assume that the parameters

$$
\begin{gathered}
A=5, \quad \alpha_{1}=0.9, \quad \alpha_{2}=0.9, \quad \mu=0.005, \quad \gamma=0.02, \\
\alpha=0.005, \quad \beta=0.1, \quad \delta=0.001
\end{gathered}
$$

are fixed and

$$
f(S, I)=\frac{\beta S I}{1+\alpha_{1} S+\alpha_{2} I}, \quad p(\tau)=\frac{1}{h} .
$$

The distributed SIRI epidemic model (1) and the discrete SIRI epidemic model (11) generate the same global asymptotic stability for certain values of $h$. However for $h \in[286,810]$ this equivalence was not true: the system (1) has the disease free equilibrium and the endemic equilibrium $\left(R_{0}>1\right)$, but the system (11) has only a disease free equilibrium $\left(R_{0} \leq 1\right)$ (see, Table 1$)$.

Table 1. Dependence of the basic reproduction number $R_{0}$ on a distributed and a discrete latent period.

\begin{tabular}{|c|c|c|}
\hline$h($ days $)$ & $R_{0}$ of a discrete latent period & $R_{0}$ of a distributed latent period \\
\hline 200 & 1.53 & 2.63 \\
\hline 285 & 1.00 & 2.22 \\
\hline 286 & 0.99 & 2.21 \\
\hline 290 & 0.98 & 2.20 \\
\hline 300 & 0.93 & 2.15 \\
\hline 819 & 0.07 & 0.99 \\
\hline
\end{tabular}

\subsection{Effect of the Relapse Rate on the Basic Reproduction Number}

The basic reproduction number $R_{0}$ is a strictly monotonically increasing function of relapse rate $\delta$ $\left(\frac{\mathrm{d} R_{0}}{d \delta}>0\right)$ (see Fig. 3). Therefore, the relapse is a factor favoring the installation of the disease in the concerned population by increasing the value of $R_{0}$ at a higher level to one. Moreover, ignoring the relapse in an epidemiological model will underestimate $R_{0}$.

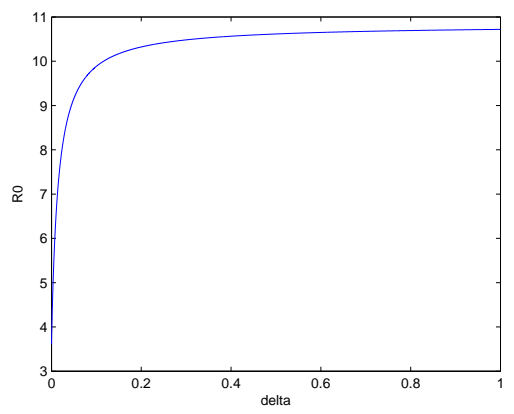

Figure 3. Dependance of the basic reproduction number on the relapse rate of the distributed SIRI epidemic model for $A=5 ; h=10 ; \alpha_{1}=0.9 ; \alpha_{2}=0.9 ; \mu=0.005 ; \gamma=0.02 ; \alpha=0.005 ; \quad$ and $\beta=0.1$ 


\section{Conclusion and Future Research}

In recent years, modeling the latent period by a distributed time delay has more attracted the attention of mathematical researchers, because the experiments and the statistical studies for some diseases have shown that this period may be modeled by distributed time delay. Moreover, the distributed delay is used in the epidemiological models to examine the cumulative effect of the incidence function.

In this work, we presented a mathematical analysis for a SIR epidemiological model with a distributed delay, a general incidence function and the relapse phenomenon (1). The originality of this work is to have a basic reproduction number $R_{0}$ which depends on the distribution of the latent period. This number remains a key parameter for the stability analysis of epidemiological models: if $R_{0} \leq 1$, then the disease free equilibrium is globally asymptotically stable and if $R_{0}>1$, then the unique endemic equilibrium is globally asymptotically stable.

In addition, we proved that: On one hand, the dynamics of the discrete SIRI model (11) is different, in some senses, to the one given by the distributed SIRI model (1) (see Fig. 1, Fig. 2 and Table 1). On the other hand, the relapse is a factor favoring the installation of the disease by increasing the value of $R_{0}$ at a higher level to one $\left(\frac{\mathrm{d} R_{0}}{d \delta}>0\right)$.

For the future research, we will consider an SIRI model with a non-constant total population, a logistic recruitment, a non-linear relapse rate and an anti-relapse treatment.

\section{References}

1. Cooke K. L., Stability analysis for a vector disease model, Rocky Mountain Journal of mathematics, Vol. 9, no. 1, pp. 31-42, (1979).

2. Beretta E. and Takeuchi Y., Global stability of an SIR epidemic model with time delays, Journal of Mathematical Biology, 33, 250-260, (1995).

3. Abta A., Kaddar A. and Talibi Alaoui H., Global Stability for Delay SIR and SEIR Epidemic Models with Saturated Incidence Rates, Electronic Journal of Differential Equations, Vol. 2012, No. 23, pp. 1-13, (2012).

4. Kaddar A., A comparison of delayed SIR and SEIR epidemic models, Nonlinear Analysis: Modelling and Control, Vol. 16, No. 2, 181-190, (2011).

5. Li G., Wang W., Wang K., Jin Z., Dynamic behavior of a parasite-host model with general incidence, J. Math. Anal. Appl., pp. 631-643, (2007).

6. Bernoussi A., Kaddar A., and Asserda S., Global Stability of a Delayed SIRI Epidemic Model with Nonlinear Incidence, International Journal of Engineering Mathematics Volume 2014, (2014).

7. Beretta E., Hara T., Ma W., and Takeuchi Y., Permanence of an SIR epidemic model with distributed time delays, Tohoku Math. J. (2), Volume 54, Number 4, 471-597, (2002).

8. Nakata Y., Enatsu Y., and Muroya Y., On the global stability of an SIRS epidemic model with distributed delays, Published in Discrete and Continuous Dynamical Systems Supplement, 1119-1128, (2011).

9. Van der Goot J.A., Koch G., De Jong M.C.M., Van Boven M., Quantification of the effect of vaccination on transmission of avian influenza (H7N7) in chickens, Proc. Natl. Acad. Sci. USA, 102, 18141-18146, (2005).

10. Eichner M., Dowell S.F., Firese N., Incubation Period of Ebola Hemorrhagic Virus Subtype Zaire, Public Health Res Perspect 2(1), 3-7, (2011).

11. Chin J., Control of Communicable Diseases Manual, American Public Health Association, Washington, (1999).

12. Martin S. W., Livestock Disease Eradication: Evaluation of the Cooperative StateFederal Bovine Tuberculosis Eradication Program, National Academy Press, Washington, (1994).

13. Tudor D., A deterministic model for herpes infections in human and animal populations, SIAM Rev. 32, pp. 136-139, (1990).

14. K.E. VanLandingham, H.B. Marsteller, G.W. Ross, and F.G. Hayden, Relapse of herpes simplex encephalitis after conventional acyclovir therapy, JAMA, 259, 1051-1053, (1988).

15. Anderson R. M., May R. M., Regulation and stability of host-parasite population interactions: I. Regulatory processes, The Journal of Animal Ecology, Vol. 47, no. 1, pp. 219-267, (1978).

16. Gabriela M., Gomes M., White L. J., Medley G. F., The reinfection threshold, Journal of Theoretical Biology, Vol. 236, pp. 111-113, (2005).

17. Jiang Z., Wei J., Stability and bifurcation analysis in a delayed SIR model, Chaos, Solitons and Fractals, Vol. 35, pp. 609-619, (2008).

18. Wang W., Ruan S., Bifurcation in epidemic model with constant removal rate infectives, Journal of Mathematical Analysis and Applications, Vol. 291, pp. 775-793, (2004). 
19. Zhang F., Li Z. Z., Zhang F., Global stability of an SIR epidemic model with constant infectious period, Applied Mathematics and Computation, Vol. 199, pp. 285-291, (2008).

20. Zhou Y., Liu H., Stability of periodic solutions for an SIS model with pulse vaccination, Mathematical and Computer Modelling, Vol. 38, pp. 299-308, (2003).

21. Chen L. S., Chen J., Nonlinear biologicl dynamics system, Scientific Press, China, (1993).

22. Wei C., Chen L., A delayed epidemic model with pulse vaccination, Discrete Dynamics in Nature and Society, Vol. 2008, Article ID 746-951, (2008).

23. Zhang J.-Z., Jin Z., Liu Q.-X., Zhang Z.-Y., Analysis of a delayed SIR model with nonlinear incidence rate, Discrete Dynamics in Nature and Society, Vol. 2008, Article ID 66153, (2008).

24. Capasso V., Serio G., A generalization of Kermack-Mckendrick deterministic epidemic model, Math. Biosci. Vol. 42, pp. 41-61, (1978).

25. Xu R., Ma Z., Stability of a delayed SIRS epidemic model with a nonlinear incidence rate, Chaos, Solitons and Fractals, Vol. 41, Iss. 5, pp. 2319-2325, (2009).

26. Kaddar A., Stability analysis in a delayed SIR epidemic model with a saturated incidence rate, Nonlinear Analysis: Modelling and Control, Vol. 15, No. 3, 299-306, (2010).

27. De Jong M.C.M., Diekmann O. and Heesterbeek H., How does transmission of infection depend on population size? In: Epidemic models: their structure and relation to data, Mollison D. (Ed.), Cambridge University Press, Cambridge, 84-94, (1995).

28. Hethcote H. W., The Mathematics of Infectious Disease, SIAM review 42, 599-653, (2000).

29. Moreira H. N., Wang Y., Global stability in an $S \rightarrow I \rightarrow R \rightarrow I$ model, SIAM Rev. 39, pp. 496-502, (1997).

30. Castillo-Garsow C., Jordan-Salivia G., Rodriguez Herrera A., Mathematical models for the dynamics of tobacco use, recovery, and relapse, Technical Report Series BU-1505-M, Cornell University, (2000).

31. Blower S., Modelling the genital herpes epidemic, Herpes 11, Suppl. 3, (2004).

32. Vargas-De-Leon C., On the global stability of infectious diseases models with relapse, Abstraction \& Application, 9, 50-61, (2013).

33. Georgescu P., Zhang H., A Lyapunov functional for a SIRI model with nonlinear incidence of infection and relapse, Applied Mathematics and Computation, 219 (2013), pp. 8496-8507.

34. Wang J., Liu X., Pang J. and Hou D., Global dynamics of a multi-group epidemic model with general exposed distribution and relapse, Osaka J. Math. 52 (2015), 117-138.

35. van den Driessche P. and Zou X., Modeling relapse in infectious diseases, Math. Biosci., 207, 89-103, (2007).

36. van den Driessche P., Wang L., Zou X., Modeling diseases with latency and relapse, Math. Biosci. Eng., 4, pp. 205-219, (2007).

37. Liu S., Wang S. and Wang L., Global dynamics of delay epidemic models with nonlinear incidence rate and relapse, Nonlinear Analysis: Real World Applications, 12, pp. 119-127, (2011).

38. Xu R., Global dynamics of an SEIRI epidemiological model with time delay, Applied Mathematics and Computation Volume 232, 1, Pages 436-444, (2014).

39. Sampath B. G. and Pradeep A., Local stability properties of a delayed SIR model with relapse effect, International Journal of Scientific and Research Publications, Volume 5, Issue 10, October 2015.

40. Beretta E. and Takeuchi Y., Global stability of an SIR epidemic model with time delays, J. Math. Biol. 33 (1995) 250-260.

41. Enatsu Y., Nakata Y., Muroya Y., Lyapunov functional techniques for the global stability analysis of a delayed SIRS epidemic model, Nonlinear Analysis: Real World Applications, Volume 13, Issue 5, October 2012, Pages 2120-2133.

42. Paulhus C. and Wang X.-S., Global stability analysis of a delayed susceptible-infected-susceptible epidemic model, Journal of Biological Dynamics, 2015 Vol. 9, Suppl. 1, 45-50.

43. Mickens R., Nonstandard Finite Difference Models of Differential Equations, World Scientific, Singapore, (1994).

44. Mickens R., Advances in the Applications of Nonstandard Finite Difference Schemes, World Scientific, Singapore, (2000).

45. Sekiguchi M. and Ishiwata E., Global dynamics of a discretized SIRS epidemic model with time delay , Journal of Mathematical Analysis and Applications , Volume 371 , pp. 195-202 (2010) 